\title{
Data Mining Techniques for Anti-Money Laundering
}

\author{
Assem Khalaf Ahmed Allam El-Din \\ computer science and information systems \\ Sadat Academy \\ Cairo, Egypt
}

\author{
Nashaat El Khamesy, PhD \\ Head of \\ computer science and information systems \\ Sadat Academy \\ Cairo, Egypt
}

\begin{abstract}
We use a data mining framework that is based on evaluating four types of neural networks, and that uses dataobtained from regular records collected from banks, to produce a classification conclusion on "who are money laundering and who are not". This will be attained by evaluating the outcomes of various types of neural networks, namely, the Multi-Layer Perceptron Neural Network (MLP), Probabilistic Neural Network (PNN), Radial Basis Function (RBF) and Linear Neural Network (LNN). Then compare these outcomes with standard statistical results. Creating by this an accurate and fast basis for decision-making which otherwise could take days or even months.
\end{abstract}

\section{Keywords}

anti-money laundering, money laundering, Electronic Crimes, Intelligent Modeling, Neural Networks, classification, artificial Neural Networks.

\section{INTRODUCTION}

Increased money laundering operations in the world as a result of the growing activity of organized crimes, and since the past few years taken the issue of money laundering and methods of combating and eradicating occupies the most prominent location in the global policy agenda and that along with the issues of (international terrorism).

Has become a money-laundering operations constitute a heavy burden on the State and became seen as a taboo legal

\section{DATA MINING CONCEPTS}

\subsection{Definition}

Data mining may be thought as "the exploration and analysis, by automated or semiautomatic means, of substantial amounts of data to discover important patterns and rules" [3]. Hence, it might be thought to be mining knowledge from considerable amounts of data as it will involve knowledge removal, as well as data pattern analysis [1].

\subsection{Tasks}

Many of the tasks suited to the application of files exploration tend to be group, evaluation, prediction, appreciation collection, clustering, along with outline. A variety of them tend to be finest approached in the top-down fashion as well as hypothesis testing although some tend to be finest approached in the bottom-up fashion termed understanding discovery often led as well as undine.

As for Classification, it is the most common data mining task and it consists of examining the features of a newly presented object in order to assign it to one of a predefined set of classes [2].

While classification deals with discrete outcomes, estimation deals with continuously-valued outcomes. In real- life cases, estimation is often used to perform a classification task [2].

Prediction deals with the classification of records according to some predicted future behavior or estimated future value. Both Affinity grouping and market basket analysis have as an objective to determine the things that can go together. Clustering aims at segmenting a heterogeneous population into a number of more homogeneous subgroups or clusters that are not predefined [12].

Description is concerned with describing and explaining what is going in a complicated database so as to provide a better understanding of the available data [12].

\subsection{Data Mining Techniques (Classification)}

In these problems, the operative is to assign each record in the database a particular class or a category label from a finite set of predefined class labels. For example, a bank would be interested in classifying each of its customers as potentially interested in a new credit card or not. All decisions involving Yes/No selection, such as classifying insurance claims according to the possibility of fraud, also belong to classification problems. Classification problems may involve three or more levels, such as "high," "medium," and "low." The main point is that the number of classes is finite. Note that there could be an implicit order relationship in the definition of the classes, such as "high," "medium," and "low." [1].

Neural Network Proposed Techniques :

\subsubsection{Multilayer Perceptrons Neural Network}

Multi-Layer perceptron (MLP) is a supply forward neural network with one or more layers between input and output layer, The first layer is called the input layer, the last one is the output layer, and in between there may be one or more hidden layers .Feed forward means that data flows in one direction from input to output layer (forward). This type of network is trained with the back propagation learning algorithm. MLPs are widely used for pattern classification, recognition, prediction and approximation. Multi-Layer Perceptron can solve problems which are not linearly separable [2].

\subsubsection{Probabilistic Neural Network:}

Probabilistic neural networks can be used for classification problems. When an input is presented, the first layer computes distances from the input vector to the training input vectors and produces a vector whose elements indicate how close the input is to a training input. The second layer sums these contributions for each class of inputs to produce as its net output a vector of probabilities. Finally, a compete transfer function on the output of the second layer picks the maximum of these probabilities, and produces a 1 for that class and a 0 for the other classes [2]. 
PNN is a useful neural network architecture with slightly different in fundamentals from back propagation. The architecture is feed forward in nature which is similar to back propagation, but differs in the way that learning occurs. PNN is supervised learning algorithm but includes no weights in its hidden layer [2].

\subsubsection{Linear Neural Network}

The linear network provides a good benchmark against which to compare the performance of your neural networks[13]. It is quite possible that a problem that is thought to be highly complex can actually be solved as well by linear techniques as by neural networks. If you have only a small number of training cases, you are probably anyway not justified in using a more complex model [2].

Here you design a linear network that, when presented with a set of given input vectors, produces outputs of corresponding target vectors. For each input vector, you can calculate the network's output vector. The difference between an output vector and its target vector is the error. You would like to find values for the network weights and biases such that the sum of the squares of the errors is minimized or below a specific value. This problem is manageable because linear systems have a single error minimum. In most cases, you can calculate a linear network directly, such that its error is a minimum for the given input vectors and target vectors [2].

\subsubsection{Radial Basis Function}

Radial basis function (RBF) networks are feed-forward networks trained using a supervised training algorithm. They are typically configured with a single hidden layer of units whose activation function is selected from a class of functions called basis functions. While similar to back propagation in many respects, radial basis function networks have several advantages. They usually train much faster than back propagation networks. They are less susceptible to problems with non-stationary inputs because of the behavior of the radial basis function hidden units [2].

The design of a RBF in its most basic form consists of three separate layers. The input layer is the set of source nodes (sensory units). The second layer is a hidden layer of high dimension. The output layer gives the response of the network to the activation patterns applied to the input layer [2].

Radial basis function neural networks (RBF) form a class of artificial neural networks, which has certain advantages over other types of neural networks including better approximation capabilities, simple network structure, and faster learning. RBF training is mainly based on the estimation of various parameters: centers, widths, connecting weights between neurons etc [2].

\section{RELATED WORK}

\subsection{Classifying Blood Donors Using Data Mining Techniques}

This study has revealed classification procedures for Blood Collection Donors data sets. This, in fact, is attained through the use of data mining classifiers to create a decision tree. The main target of this study is the advancement of a system that is required for the timely evaluation of huge Blood Group Donors data sets.

\subsection{Money Laundering Crime under Electronic Payment Background}

As the result of information technology's development, promoted the tradition payment means enormously to the computerization development advancement. The electronic payment has accelerated the money circulation speed, and has brought the enormous convenience for the modern trade activity[4]. But the electronic payment's characteristics such as invisible, high speed, concealment and so on have provided the enormous convenience to the money laundering criminal activity, and presented the enormous challenge to the traditional counter- money laundering system[9].

\subsection{Neural Networks In Data Mining}

Companies have been collecting data for decades, building massive data warehouses in which to store it. Even though this data is available, very few companies have been able to realize the actual value stored in it. The question these companies are asking is how to extract this value. The answer is Data mining. There are many technologies available to data mining practitioners, including Artificial Neural Networks, Regression, and Decision Trees. Many practitioners are wary of Neural Networks due to their black box nature, even though they have proven themselves in many situations. This paper is an overview of artificial neural networks and questions their position as a preferred tool by data mining practitioners[15].

\subsection{Data Mining-Based Solution For Detecting Suspicious Money Laundering Cases In An Investment Bank}

Money laundering poses a serious threat not only to financial institutions but also to the nation. This criminal activity is becoming more and more sophisticated and seems to have moved from the cliché of drug trafficking to financing terrorism and surely not forgetting personal gain[6]. Most international financial institutions have been implementing anti-money laundering solutions to fight investment fraud. However, traditional investigative techniques consume numerous man-hours. Recently, data mining approaches have been developed and are considered as well-suited techniques for detecting money laundering activities[8].

\section{PROPOSED SOLUTION}

\subsection{Proposed Framework and Case Investigation for Problem Solving}

The actual Framework of research shows in Fig.1. That the Data Mining applied the thinking ability issue solving. This will be attained by evaluating various types of neural networks[11], the Multi-Layer Perceptron Neural Network (MLP), Probabilistic Neural Network (PNN), Radial Basis Function (RBF) and Linear Neural Network (LNN) to a regular record outcome that collected from banks [10], and selecting the neural network giving the highest functionality in facts classification to to produce a conclusion on "who are money laundering and who doesn't". 


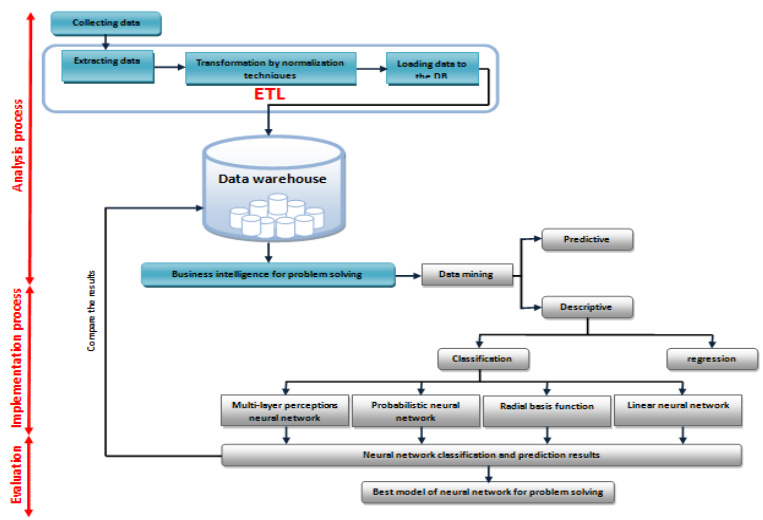

Fig. 1. Proposed framework for problem solving

\subsection{The Framework Procedure:}

\subsection{1 collecting data "Source of Data"}

- Bank Sheets.

Method of Data Collection

- Appointments with bank officials (IT Department) in bank.

- Using bank paperwork (paper and USB drives).

- $\quad$ Taking notes.

\subsubsection{Data Extraction}

With this research we all collected instances from the money laundry coming from banks. Lots of data are usually determined by one another; the data repeat will give a mistake as well as low classification performance within the attained phase. The advices of neural network should be impartial variables. This method of taking out the impartial data for problem solving in the huge data compiled.

\subsubsection{Transformation Process}

The actual data: including data purchased from your bank, are usually be imperfect, noisy and inconsistent, data cleanup work to fill up on missing valuations and proper in congruencies in the data [10]. The change process will start by producing an ERP Schema, which usually changes 150 unstructured paperwork to methodized report.

\subsubsection{Missing value:}

Many etiquette solve this matter based on the volume of this missing values and the quantity of its reliance in the concluding decision. Therefore many of us load the lacking valuations personally while using the continuous value.

\subsubsection{Noisy data:}

Can be a arbitrary mistake in calculated valuations there are lots of etiquette to miss the disturbance data much like to clustering. We are able to show the noisy data by comparable ideals structured to class after that we could consider the outliers data and manage it physically[5].

\subsubsection{The Inconsistent data:}

You will find contradictions from the data which take place for many transactions; there are several data there are many repaired personally making use of additional recommendations. Some other contradictions data happen because of the data integration.

As an example presented feature features distinct names in several database, redundancy could also can be found.

\subsubsection{The Data Integration:}

By different data options in to one data keep referred to as (Data Warehouse). These data sources employ a several approach to get into the data in one data retail store just like a smooth document, and a several database, as an example, case_id in one more database termed customer_id and so on. Cautious integration of the data from several resources helps in minimizing and preventing redundancies and disparity in the caused data set. This can help enhancing the accuracy and reliability and rate from the following mining process.

\subsubsection{The Data Selection:}

Choosing areas of data of specific fascination for the lookup area is the simplest way to acquire final results highly relevant to the lookup standards. On this study many of us choose the unbiased changing impact acquiring the money laundry instances as suggestions, including the quantity of the economic transactions, the size of the personal transactions, the typical annual revenue of the consumer, categorization of the consumer, level of the consumer, and the cash flow options. However choose the reliant factors as result, which can be your decision considered "if the client is committing money laundry or not" This choice will improve the neural network performance effectiveness

\subsubsection{Data Transformation:}

The Data transformed while using Normalization method is especially helpful for classification algorithms including neural networks[14]. Normalizing the insight valuations for each and every characteristic calculated in the teaching will assist you to improve the learning phase [10]. The normalization method transactions the data in to appropriate forms for the mining procedure, the place that the characteristic data are scaled so as to tumble inside a small specific selection.

\section{TARGET DATA SET}

The actual study applied $70 \%$ of data intended for teaching the neural network and $30 \%$ will probably be applied as a totally impartial check on the network. And that's due to the fact, if the neural network has been analyzed for a passing fancy pair of data which were useful for the education, it won't be distinct temperature it discovered to "predict" or to "memorize" patterns.

TABLE 1. Sample of data Input and the factors affecting the database after normalization process

\begin{tabular}{|c|c|c|c|c|c|c|c|c|c|c|}
\hline \multicolumn{11}{|c|}{ Independent Factors affecting on customers } \\
\hline$\#$ & $\begin{array}{l}\text { transaction } \\
\text { Count }\end{array}$ & $\begin{array}{l}\text { Transaction } \\
\text { Iỵpe }\end{array}$ & $\begin{array}{c}\text { Transaction } \\
\text { Amount }\end{array}$ & $\begin{array}{c}\text { Transaction } \\
\text { Country }\end{array}$ & $\begin{array}{l}\text { Customer } \\
\text { Location }\end{array}$ & $\begin{array}{l}\text { Customer } \\
\text { Work } \\
\text { lerel }\end{array}$ & $\begin{array}{l}\text { Customer } \\
\text { Type }\end{array}$ & $\begin{array}{l}\text { Customer } \\
\text { Other } \\
\text { Income }\end{array}$ & $\begin{array}{l}\text { Customer } \\
\text { Categgory }\end{array}$ & $\begin{array}{l}\text { Customer } \\
\text { Decision }\end{array}$ \\
\hline 1 & 0.1818 & 0.869565 & 0 & 0 & 0.3333 & 1 & 1 & 1 & 1 & 0 \\
\hline 2 & 0.1818 & 0.860565 & 0 & 0 & 0.3333 & 0.0606066 & 1 & 1 & 1 & 0 \\
\hline 3 & 0.1818 & 0.860565 & 0 & 0 & 0.3333 & 1 & 1 & 1 & 1 & 0 \\
\hline 4 & 0.1818 & 0.860565 & 0 & 0 & 0.3333 & 1 & 1 & 1 & 1 & 0 \\
\hline 5 & 0.1818 & 0.869565 & 0 & 0 & 0.3333 & 1 & 1 & 1 & 1 & 0 \\
\hline 6 & 0.1818 & 0.869565 & 0 & 0 & 0.3333 & 0.0606066 & 0 & 1 & 0.75 & l \\
\hline 7 & 0.1818 & 0.8605605 & 0 & 0 & 0.3333 & 1 & 1 & 1 & 1 & 0 \\
\hline 8 & 0.1818 & 0.434782 & 0 & 0 & 0.3333 & 1 & 1 & 0 & 1 & 0 \\
\hline 9 & 0.1818 & 0.434782 & 0 & 0 & 0.3333 & 1 & 1 & 1 & 1 & 0 \\
\hline 10 & 0.1818 & 0.434782 & 0 & 0 & 0.3333 & 0.066066 & 1 & 1 & 1 & 0 \\
\hline
\end{tabular}

When the number of layers, as well as number of items in every layer, are already chosen, the network's weight and thresholds should be arranged to reduce the conjecture mistake produced by the networks. Here is the function of the teaching algorithms. The traditional instances which have been collected are employed to instantly modify the weight load and to be able to reduce this mistake. 


\section{PROBLEM SOLUTION}

The STATISTICA is a thorough, included data analysis, visuals, database management, and personalized application advancement system having a large selection of standard and innovative analytic techniques for business, data mining, science, and architectural applications.

The issue of identifying the number of neural networks layer and the number of nodes in each and every layer, which makes a lot of permutations amounting to be able to 300 networks by training and testing each and every techniques, and select the right functionality based on a stability mistake versus selection with 4 maintained networks.

\section{CLASSIFICATION PERFORMANCE}

The classification pace of the MLP, PNN, RBF, Linear Neural Network techniques are in comparison, for acknowledged factors that neural networks supply the powerful of classifying the data imports[12]. The right classification pace, the mistake pace for every neural network and the length time required for classification are revealed in Table 2.

Though the linear allows greater performance outcome together with 0.80 correct data classification followed by MLP with 0.68 and rapprochement the PNN and the RBF with 0.64 for the first one and 0.61 for the second. Though the Linear Neural Network results supply the most competitive classification mistake throughout the implantation with 0.36 normally the RBF provides the highest classification mistake with 0.58 . The MLP requires the longest time to educate the neural network and classify the data with 4 minutes and 25 seconds, while the rest of neural networks take 6 seconds to do this task.

TABLE 2. Neural Network Classification Performance in Implementation Process

\begin{tabular}{|c|l|c|c|c|}
\hline \multicolumn{5}{|c|}{ Neural Networks Classification Performance } \\
\hline$\#$ & \multicolumn{1}{|c|}{$\begin{array}{c}\text { Neural } \\
\text { Networks Type }\end{array}$} & $\begin{array}{c}\text { Correct Classification } \\
\text { Rate }\end{array}$ & $\begin{array}{c}\text { Error } \\
\text { Classification Rate }\end{array}$ & $\begin{array}{c}\text { Classification } \\
\text { Time }\end{array}$ \\
\hline $\mathbf{1}$ & Multi-Layer Perception & 0.68 & 0.39 & $4: 25$ \\
\hline $\mathbf{2}$ & ProbabilisticNeural Network & 0.64 & 0.43 & $0: 06$ \\
\hline $\mathbf{3}$ & Radial Basis Function & 0.61 & 0.58 & $0: 06$ \\
\hline $\mathbf{4}$ & Linear Neural Netwotk & 0.80 & 0.36 & $0: 06$ \\
\hline
\end{tabular}

The Linear Neural Network technique commence with 2 layer; 11 inputs, 1 output, without hidden layer, shown in Figure 5.1 .

That could resolute the 0.99 teaching functionality, 1.00 choosing efficiency and 1.00 testing efficiency and it have a 0.000001 training mistake, 0.04 select mistake and the lowest testing mistake with 0.00 , that shows in Table 3 .

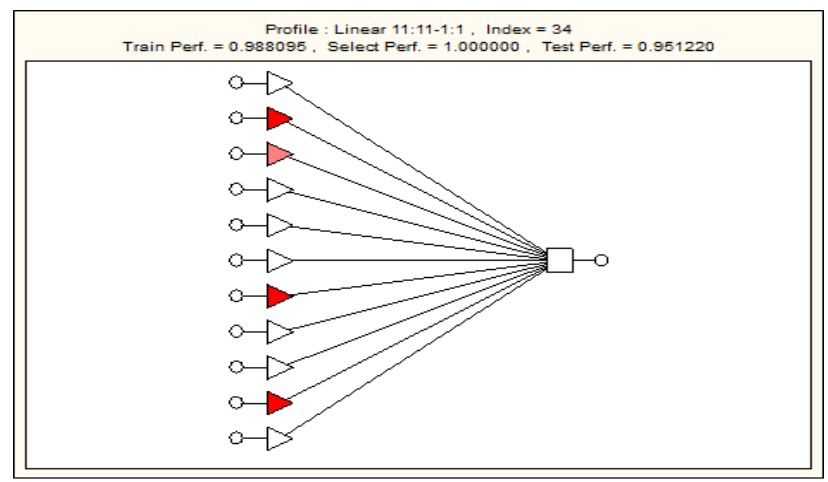

Fig. 2. Linear Neural Network Model
Outcome executed by the MLP design that was recommended by smart difficulty solver has been observed with 4 layers; 11 inputs, 1 output, and 12 hidden neurons in layer1 as well as 11 hidden neurons additionally in layer 2, shown in Figure 5.2, established the 0.98 teaching functionality, 1.00 choosing efficiency and 0.95 testing performance. On the other hand use 0.00259 selecting mistakes, 0.09 training mistakes and testing mistakes of 0.23 , which shows in Table3.

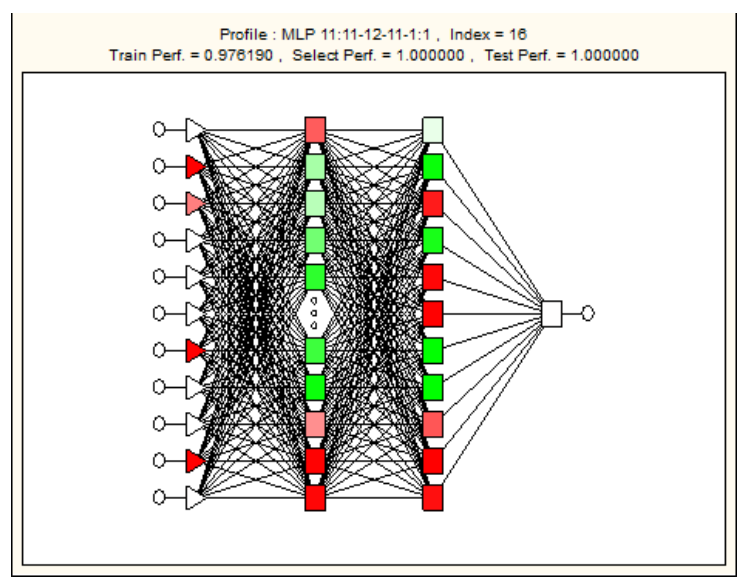

Fig. 3. MLP Neural Network Model that performed the optimal solution for problem solving

An additional solution with regard to difficulty handling was a Probabilistic Neural Network (PNN) establish with 4 layers; 11 inputs, 1 output, and two hidden layers with 84 neurons in the first hidden layer and 2 neurons in the second layer, as shown in Figure 5.3, that established the 1.00 teaching functionality, 0.98 selecting performance and 0.86 testing functionality. On the other hand having the highest teaching error with $0.99,0.99$ choose mistake and the testing error of 0.94 that are shown in Table 3.

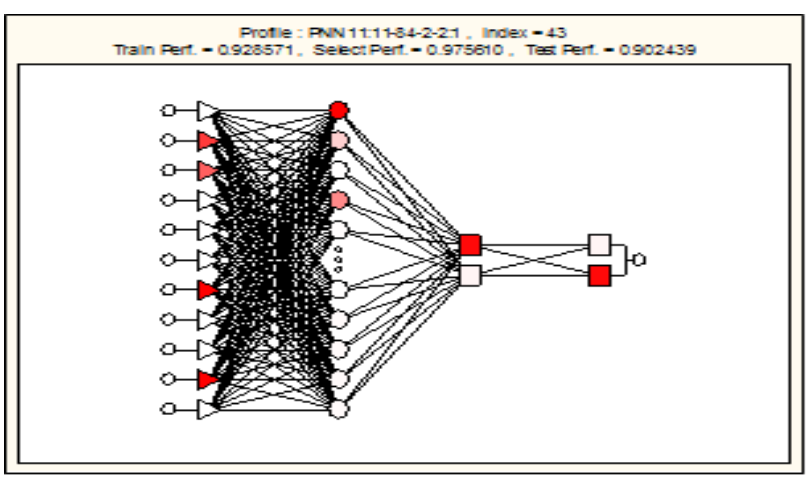

Fig. 4. PNN Neural Network Model

The Radial Basis Function (RBF) Rating as second neural network becomes an excellent outcome recognized with 5 layer; 11 inputs, 1 output, and one hidden layer with 5 neurons, shows in Figure 5.4, established the 0.65 teaching functionality, 0.80 choosing functionality and 0.73 testing functionality. On the other hand have a 0.30 training error, 0.19 select errors and a testing error of 0.27 , which are shown in Table 3. 


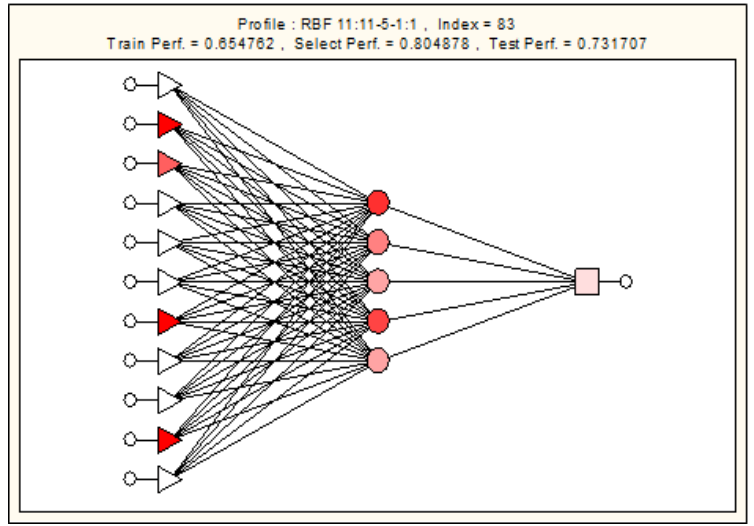

Fig. 5. The best result Radial Basis Function obtains ahighest performance:

TABLE 3. Profiling Performance of Neural Networks in Training Process

\begin{tabular}{|c|c|c|c|c|c|c|c|c|c|c|}
\hline Inder & Profile & $\begin{array}{l}\text { Train } \\
\text { Perf }\end{array}$ & $\begin{array}{l}\text { Select } \\
\text { Perf }\end{array}$ & Test Perf & \begin{tabular}{|l|} 
Train \\
Error \\
\end{tabular} & \begin{tabular}{|l|} 
Selett \\
Error \\
\end{tabular} & \begin{tabular}{c|c|} 
Test \\
Error
\end{tabular} & Inputs & Hidden(I) & Hidden(? \\
\hline & Linas 11:11:-1:1 & 0988095 & 1000000 & 1000000 & 0.000001 & \begin{tabular}{|l|l|l|l|l|l}
0.4244 \\
\end{tabular} & 0.000000 & 11 & 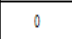 & 0 \\
\hline & 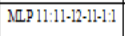 & 0976190 & 1.000000 & 0.951220 & $|0.087761|$ & 0.000259 & \begin{tabular}{|l|l|}
0260089 \\
\end{tabular} & 11 & 1 & 11 \\
\hline & & 1.000000 & 0.975610 & 0.878049 & 0.998677| & \begin{tabular}{|l|l|}
0986978 \\
\end{tabular} & 0.938239 & 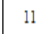 & & 2 \\
\hline & $\operatorname{RBF} 11: 11.5-51:$ & 0.654762 & 0.804878 & 0.731707 & 0295292 & \begin{tabular}{|l|}
0.194382 \\
\end{tabular} & 0269715 & 11 & 5 & 0 \\
\hline
\end{tabular}

describes the assessment involving the four forms of neural networks clarify the training, selecting, testing performance and the error rate throughout the implementation process.

\section{PREDICTION RESULTS}

TABLE 4. Shows the evaluation between the real selection result from database and the Neural Networks Prediction results, the MLP shows the best prediction results

TABLE 5. Sample of Comparison the Prediction Results of Neural Networks

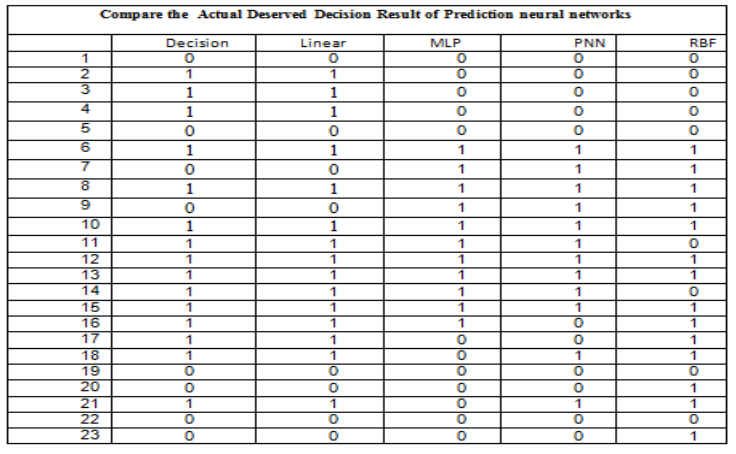

\section{SENSITIVITY ANALYSIS}

The sensitivity analysis suggests that input variable is is recognized as most critical by the Neural Network models. The tenderness is the ratio between the mistakes together with omission, the baseline mistake and ranks the factors in the order worth focusing on, and the impact on people who commit money laundry. It is shown in Table 5.
TABLE 6. Summary Reports of Neural Network Ranking Affecting Factors according of its impact

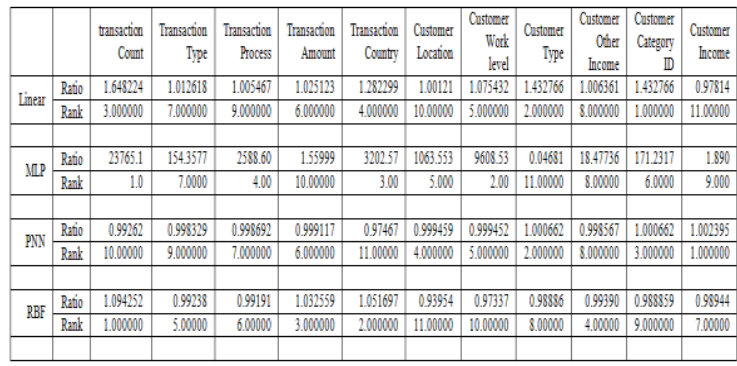

Almost all Neural Network models in comparison for difficulty solving as well as rating the elements impact the customers who make money laundry based on the impression of each one element. All models are in keeping with the wad of cash of each customer and the category of the customer is the most crucial element affecting how to determine money laundry instances. The study outcomes explain to if the money laundry ailments applied on any customer, then he would turn into a person dedicated money laundry and we should review all his thought transactions and his motions inside the bank during the period of the money laundry[7].

\section{CLASSIFICATION RESULT}

TABLE 7. Comparison of the Classification results of Neural Netw orks Profiling the Correct and the Wrong execution

\begin{tabular}{|l|l|l|l|l|l|l|l|l|}
\hline & \multicolumn{2}{|c|}{$\begin{array}{c}\text { MLP committing } \\
\text { money laundry } \\
\text { decision. }\end{array}$} & \multicolumn{2}{|c|}{$\begin{array}{c}\text { PNN committing } \\
\text { money laundry } \\
\text { decision. }\end{array}$} & \multicolumn{2}{c|}{$\begin{array}{c}\text { RBF committing } \\
\text { money laundry } \\
\text { decision. }\end{array}$} & \multicolumn{2}{|c|}{$\begin{array}{c}\text { Linear committing } \\
\text { money laundry } \\
\text { decision. }\end{array}$} \\
\hline $\begin{array}{c}\text { Classification } \\
\text { Factors }\end{array}$ & Committed & $\begin{array}{r}\text { Didn't } \\
\text { commit. }\end{array}$ & Committed & $\begin{array}{c}\text { Didn't } \\
\text { commit }\end{array}$ & Committed & $\begin{array}{r}\text { Didn't } \\
\text { commit }\end{array}$ & Committed & $\begin{array}{c}\text { Didn't } \\
\text { commit }\end{array}$ \\
\hline Total & 149.0000 & 17.00000 & 149.0000 & 17.00000 & 149.0000 & 17.00000 & 149.0000 & 17.000000 \\
\hline Correct & 149.0000 & 15.00000 & 147.0000 & 13.00000 & 104.0000 & 14.00000 & 149.0000 & 14.000000 \\
\hline Wrong & 0.0000 & 2.00000 & 2.0000 & 4.00000 & 45.0000 & 3.00000 & 0.0000 & 3.00000 \\
\hline Unknown & 0.0000 & 0.00000 & 0.0000 & 0.00000 & 0.0000 & 0.00000 & 0.0000 & 0.00000 \\
\hline Correct (\%) & 100.0000 & 88.23529 & 98.6577 & 76.47059 & 69.7987 & 82.35294 & 100.0000 & 82.35294 \\
\hline Wrong (\%) & 0.0000 & 11.76471 & 1.3423 & 23.52941 & 30.2013 & 17.64706 & 0.0000 & 17.64706 \\
\hline Unknown (\%) & 0.0000 & 0.00000 & 0.0000 & 0.00000 & 0.0000 & 0.00000 & 0.0000 & 0.00000 \\
\hline
\end{tabular}

The actual Classification Evaluation outcomes of Neural Networks, which profiling the correct and the wrong performance shows that the MLP provides the best classification rate in Table 6 .

\section{CONCLUSIONS}

The LNN neural network gives the best performance of classification rate and high performance of training, selecting and testing the data and lowest error.

The Linear Neural Network (LNN) Neural Network approach could be performed on other financial transactions according to multi factors and many other applications.

The research helps you to slow up the time of data evaluation and enhances the pace of conclusion creating, and the recourses employed for evaluation method like paper, document as well as file. Additionally there is much you can 'teach' the actual neural networks by serving it data so as to get exact outcomes and powerful.

\section{REFERENCES}

[1] Gordon S ,Linoff.,Michael,J.,Berry Apr.2011 Data Mining Techniques: For Marketing, Sales, and Customer Relationship Management, Wiley Computer Publishing; 3rd. 
[2] Jiawei,Han.,Kamber,Micheline.,Pei,Jian.,Mar 2011 Data Mining Concepts and Techniques 3rd,Morgan Kaufmann Publishers.

[3] Ying,Huang.,Bing,Quan.,Huang,Tahar,M.,Kechadi,2011 A Rule-Based Method for Customer Churn Prediction in Telecommunication Services, Springer,pp.411-422.

[4] Peng,Weibing.,Jul.2011 Research on Money Laundering Crime under Electronic Payment Background. Journal of Computers, Vol. 6, No. 1.

[5] NhienAn,LeKhac.,Martin,Bue.,Michael,Whelan.,Tahar, M.,Kech,adi. 2010 A Clustering-Based Data Reduction for Very Large Spatio-Temporal Datasets, Springer,pp.43-54

[6] Nhien-An,Le-Khac.,Sammer,Markos.,Tahar-Kechadi,M., 2010 A Data Mining-Based Solution for Detecting Suspicious Money Laundering Cases in an Investment Bank, pp. 235-240 .

[7] Nhien-An,Le-Khac., Tahar-Kechadi,M., 2010 Application of Data Mining for Anti-money Laundering Detection: A Case Study, ICDM Workshops: pp.577-584 -

[8] Nhien-An,Le-Khac.,Sammer,Markos.,Mohand,TaharKechadi. 2010 Performance Evaluation of a Knowledgebased Solution for Detecting Money Laundering in an Investment Bank, Proceedings of the International Conference on Information \& Knowledge Engineering, pp.57-63.
[9] Nhien-An,Le-Khac.,Sammer,Markos.,Mohand,TaharKechadi., 2009 Towards a New Data Mining-Based Approach for Anti-Money Laundering in an International Investment Bank, Springer, pp. 77-84.

[10] Nhien-An,Le-Khac.,Sammer,Markos.,Michael,O'Neill., Anthony,Brabazon.,Tahar-Kechadi,M.,2009 An Efficient Search Tool For An Anti-Money Laundering Application Of An Multi-National Bank's Dataset, Proceedings of the 2009 International Conference on Information \& Knowledge Engineering,pp.151-157.

[11] S.Xu,Zhang,M.,2005 Data mining - an adaptive neural network model for financial analysis, Information Technology and Applications, IEEE, ICITA, pp.336-340.

[12] Rabunal,J., Dorado,J., 2006 Artificial neural networks in real-life applications, pp.297-303.

[13] X,Ni.,2008 Research of Data Mining Based on Neural Networks, World Academy of Science, Engineering and Technology.

[14] Singh,Y.,Singh-chauha,A.,2009 NEURAL NETWORKS IN DATA MINING, Journal of Theoretical and Applied Information Technology.

[15] Rabunal ,J., Dorado,J., 2006 Artificial neural networks in real-life applications, IDEA group publish, pp.297303. 